\title{
AN IMPROVED UPPER BOUND ON NEIGHBOR EXPANDED SUM DISTINGUISHING INDEX
}

\author{
BOJAN VUČKOVIĆ \\ Mathematical Institute, Serbian Academy of Science and Arts, \\ Kneza Mihaila 36 (P.O. Box 367), 11001 Belgrade, Serbia \\ e-mail: b.vuckovic@turing.mi.sanu.ac.rs
}

\begin{abstract}
A total $k$-weighting $f$ of a graph $G$ is an assignment of integers from the set $\{1, \ldots, k\}$ to the vertices and edges of $G$. We say that $f$ is neighbor expanded sum distinguishing, or NESD for short, if $\sum_{w \in N(v)}(f(v w)+f(w))$ differs from $\sum_{w \in N(u)}(f(u w)+f(w))$ for every two adjacent vertices $v$ and $u$ of $G$. The neighbor expanded sum distinguishing index of $G$, denoted by egndi ${ }_{\sum}(G)$, is the minimum positive integer $k$ for which there exists an NESD weighting of $G$. An NESD weighting was introduced and investigated by Flandrin et al. (2017), where they conjectured that egndi $\sum_{\Sigma}(G) \leq 2$ for any graph $G$. They examined some special classes of graphs, while proving that $\operatorname{egndi}_{\sum}(G) \leq \chi(G)+1$. We improve this bound and show that egndi $_{\sum}(G) \leq 3$ for any graph $G$. We also show that the conjecture holds for all bipartite, 3-regular and 4-regular graphs.
\end{abstract}

Keywords: general edge coloring, total coloring, neighbor sum distinguishing index.

2010 Mathematics Subject Classification: 05C15.

\section{REFERENCES}

[1] G. Chartrand and P. Zhang, Chromatic Graph Theory (Boca Raton: Chapman \& Hall/CRC, 2009).

[2] E. Flandrin, H. Li, A. Marczyk, J.-F. Sacle and M. Woźniak, A note on neighbor expanded sum distinguishing index, Discuss. Math. Graph Theory 37 (2017) 29-37. doi:10.7151/dmgt.1909

[3] M. Karoński, T. Luczak and A. Thomason, Edge weights and vertex colours, J. Combin. Theory Ser. B 91 (2004) 151-157.

doi:10.1016/j.jctb.2003.12.001 
[4] M. Kalkowski, M. Karoński and F. Pfender, Vertex-coloring edge-weightings: Towards the 1-2-3-conjecture, J. Combin. Theory Ser. B 100 (2010) 347-349. doi:10.1016/j.jctb.2009.06.002

[5] R.L. Brooks, On coloring the nodes of a network, Proc. Cambridge Philos. Soc. 37 (1941) 194-197. doi:10.1017/S030500410002168X

Received 16 February 2017

Revised 19 March 2018

Accepted 19 March 2018 